\title{
BMJ Open Psychometric properties of a self- assessment questionnaire concerning symptoms and impairment in urinary tract infections: the UTI-SIQ-8
}

\author{
Ildikó Gágyor (D) , ${ }^{1,2}$ Katrin Rentzsch (D) ,, Stephanie Strube-Plaschke (D) ,2 \\ Wolfgang Himmel (D) ${ }^{2}$
}

To cite: Gágyor I, Rentzsch K, Strube-Plaschke S, et al. Psychometric properties of a self-assessment questionnaire concerning symptoms and impairment in urinary tract infections: the UTI-SIQ-8. BMJ Open 2021;11:e043328. doi:10.1136/ bmjopen-2020-043328

- Prepublication history and additional material for this paper are available online. To view these files, please visit the journal online (http://dx.doi. org/10.1136/bmjopen-2020043328).

IG and KR contributed equally.

Received 01 August 2020 Revised 12 December 2020 Accepted 26 January 2021

Check for updates

(C) Author(s) (or their employer(s)) 2021. Re-use permitted under CC BY-NC. No commercial re-use. See rights and permissions. Published by BMJ.

For numbered affiliations see end of article.

Correspondence to Professor Katrin Rentzsch; k.rentzsch@phb.de

\section{ABSTRACT}

Objectives To validate the urinary tract infectionSymptom and Impairment Questionnaire (UTI-SIQ-8), a questionnaire that consists of four items to assess the symptom severity for dysuria, urgency, frequenc, and low abdominal pain and four items to assess the resulting impairment of activity by UTIs.

Design Prospective observation study.

Setting German primary care practices.

Participants An unselected population of women with UTI. Women could participate online via a web application for smartphones, smartwatches and tablets or use a paper-and-pencil version.

Main outcomes Psychometric properties of the UTI-SIQ-8 regarding reliability, validity and sensitivity to change by using factor analysis and multilevel and network analysis.

Results Data from 120 women with a total of 769 symptom reports across 7 days of measurement were analysed. The majority of the participating patients (87/120) used the web application via smartphones or other devices. The reliability of the UTI-SIQ-8 was high, with Cronbach's alpha of .86 at intake; convergent and discriminant validity was satisfactory. Intraclass correlation demonstrated high sensitivity to change, with $68 \%$ of the total variance being due to time differences. These daily changes in an individual's symptoms moved parallel with daily changes in the $E Q-5 D-5 L(b=1.68$, $\mathrm{SE}=0.12, p<0.001)$ and the visual analogue scale $(b=0.03$, $\mathrm{SE}=0.003, \mathrm{p}<0.001)$, also highlighting convergent validity with respect to daily changes in symptom severity. Conclusions The present findings support the UTISIQ-8 questionnaire as an economic, reliable and valid instrument for the assessment of symptom severity and symptom change in women with uncomplicated UTI. The web application helped patients to report symptoms on a daily basis. These findings may encourage primary care physicians to use the UTI-SIQ-8 in their daily practice and researchers to apply it to studies involving patients with uncomplicated UTI.

\section{BACKGROUND}

Urinary tract infections (UTIs) are common in primary care and encompass a considerable burden of symptoms for affected patients. ${ }^{12}$ UTI occurs in women more often
Strengths and limitations of this study

- As we followed patients in their daily life on a dayto-day basis, our study is highly ecologically valid, which makes the urinary tract infection-Symptom and Impairment Questionnaire (UTI-SIQ-8) relevant for primary care physicians to use it in their daily practice and researchers to apply it to studies involving patients with uncomplicated UTI.

- The diary design of this study and the use of a web app allowed us to collect more than 750 data entries throughout the study highlighting the statistical power of our analyses.

- Using network analyses is novel in UTI research and may give direction to future research for validating self-report questionnaires.

- The UTI-SIQ-8 questionnaire is deliberately kept short to ensure a feasible implementation in daily use.

- At present, we do not advise physicians to use the UTI-SIQ-8 for individual assessment because norm values or reference values are not yet available.

than men and the typical signs are urgency, frequency and dysuria, often accompanied by low abdominal or back pain, smelly urine, haematuria and pyuria. ${ }^{34}$ From the women's perspective, particularly concerning symptom severity, the impairment and the impact on daily activities are of high relevance. ${ }^{45}$

While the initial symptoms of a UTI are essential for diagnosis, the course of symptoms, especially in the first few days, is essential to evaluate the clinical effectiveness of treatment. Accordingly, randomised controlled trials (RCTs) in this discipline use questionnaires to inform about the severity of the symptoms at onset and along the course of a UTI. ${ }^{6-10}$ For example, Ferry et al ${ }^{11}$ used frequency, urgency, dysuria and suprapubic pain as the most important patient-reported outcomes to assess success in their therapy study. 
There are a couple of symptom questionnaires or diaries for UTI in use, ${ }^{12-15}$ most of them in English, but some were translated and validated in other languages, such as Norwegian, ${ }^{6}$ Chinese ${ }^{16}$ or German. ${ }^{717}$ Most questionnaires were developed for and validated in RCTs; however, transferability in usual care may be limited due to a selection process in RCTs. Some of the symptom questionnaires focused on the severity of UTI symptoms only, ${ }^{6811}$ while others considered the impact of UTI on everyday activities. ${ }^{13}$ Some validation studies were concerned with both aspects, ${ }^{18}$ and a Danish validation study of a conditionspecific diary suggested to distinguish between severity, bothersomeness and impact on daily activities ${ }^{15}$ but only a handful assessed a questionnaire's sensitivity to change apart from an proper diagnosis. ${ }^{17}{ }^{19}$ With a proper assessment of symptoms as well as responsiveness or sensitivity to change, the urinary tract infection symptom assessment (UTISA) ${ }^{12}$ and the current Acute Cystitis Symptom Score (ACSS) Questionnaire ${ }^{18}$ appeared to be well suited for primary care patients. The authors of both expected the participating women to fill in a rather large questionnaire, comprising 14 or 18 items. The UTISA had to be completed at baseline and then at 3-hour and 8-hour intervals until all UTI symptoms were resolved.

In clinical settings; however, there is a clear need to lower the response burden on patients, especially to when the course of the disease should be followed up daily. Moreover, modern surveillance technologies increase the applicability of questionnaires. ${ }^{20}$ Following the symptoms as suggested by Ferry et al. ${ }^{11}$ and other researchers, we developed a short questionnaire comprising dysuria, frequency, urgency and suprapubic pain, with the possibility for women to assess both severity of UTI symptoms and impairment by these symptoms (bothersomeness), as suggested, among others, by Clayson $e t a l^{12}$ We have used this new questionnaire in two RCTs; $;{ }^{521}$ however, it has not been validated in the targeted population yet.

Hence, the present study aimed to validate this short self-report questionnaire to measure symptom severity and impairment by UTI and its course-called the UTI Symptom and Impairment Questionnaire (UTI-SIQ-8) in an unselected population of women with UTI in German primary care. An additional objective was to prove the applicability of a web application among participating women.

\section{METHODS}

The study was performed from November 2016 to May 2018 and comprised the following steps ${ }^{1}$ : questionnaire development, ${ }^{2}$ compilation of additional questionnaires and measuring tools for validation purposes, ${ }^{3}$ sample size calculation, ${ }^{4}$ recruitment of participants, ${ }^{5}$ data collection and $^{6}$ statistical analyses.

\section{Development of the UTI-SIQ-8 questionnaire}

The development process involved in its early stage a multiprofessional team of general practitioners, young doctors, psychiatrists, social scientists and study nurses. First, we chose outcome criteria used by Ferry $e t a l^{11}$ in their therapy study and by Clayson $e \mathrm{al}^{12}$ in their validation study and which women reported in the survey of Colgan et al. ${ }^{22}$ that is, frequent urination, urgency in urination, pain during urination (dysuria) and pain/pressure in lower stomach (abdominal pain). Following Clayson et $a l .,{ }^{12}$ we found it important to ask women for severity and impairment by these symptoms in daily life (bothersomeness). Then the multiprofessional team discussed the wording, order of the questions and the answer scores to come to relevant and clear items for women suffering from UTI. We used this version in two RCTs ${ }^{521}$ where the questionnaire proved to be feasible.

The final UTI-SIQ-8 questionnaire consists of four items to assess the symptom severity for dysuria, urgency, frequency and low abdominal pain. These items were scored from 1 (no symptoms at all) to 5 (very strong symptoms); four items assess the impact of these symptoms (bothersomeness), scored from 1 (no impairment at all) to 5 (very strong impairment). The questionnaire (and an English translation) can be seen in online supplementary appendix 1 .

\section{Compilation of questionnaires and tools for validation purposes}

To validate the UTI-SIQ-8 questionnaire, we chose the following questionnaires and tools:

\section{King's Health Questionnaire, German version}

The King's Health Questionnaire (KHQ) is a selfadministered questionnaire designed to assess the impact of urinary incontinence on quality of life. The measure was originally designed for use in women and contains 32 questions, which are scored in 10 domains. Weighted summary scores in each domain range from 0 to 100 , with higher scores indicating greater impairment. The KHQ has been validated for use in assessing women with urinary problems, such as an overactive bladder. ${ }^{23} \mathrm{~A}$ German version (KHQ_G) has also been validated. ${ }^{24}$

\section{EQ-5D-5L}

We used a validated German version of the EQ-5D-5L (hereafter abbreviated as EQ-5D), which evaluates the generic quality of life and comprises five dimensions (5D), each describing a different aspect of a patient's health status: mobility, self-care, usual activities, pain/ discomfort and anxiety/depression, with a 5-level (5L) answer format. ${ }^{25}$

\section{Visual analogue scale for pain}

We used a simple numeric-geometric scale (visual analogue scale (VAS)) to assess pain. It was developed and validated for use in clinical practice. ${ }^{26}$

\section{Sample size calculation}

One main criterion for the validation of our questionnaire was its sensitivity to change. We first calculated a sample size of more than 200 patients to have enough statistical 
power for latent variable models to detect change. Since it proved difficult to reach such a sample size in busy primary care practices, we made use of multilevel models on basis of a high number of data entries and manifest variables that ensured the statistical power of our analysis to detect change.

\section{Participants}

We invited 298 primary care physicians (ie, general practitioners and community gynaecologists) in Germany to achieve a sample of approximately 20 practices. Community gynaecologists are besides general practitioners the most often consulted physicians for UTI in German primary care. ${ }^{27}$ All participating physicians provided written informed consent.

Participating patients were included using convenient sampling. This method seemed to be practically applicable in daily routine. Patients were selected by the responsible medical assistants and physicians according to the defined inclusion and exclusion criteria. Women, 18 years and older, with a suspected uncomplicated UTI (with dysuria, urgency, frequency and low abdominal pain), were invited to participate. Women with signs of a complicated infection were not included. Participants, who gave informed consent, received an electronic code that allowed them to access a daily electronic questionnaire for the following 7 days. For more details, refer to Gágyor et al. ${ }^{28}$

Screening lists were used to calculate the participation rate; however, these lists proved not to be reliable because, due to the time pressure in day-to-day business, practice nurses had, due to practice stress, difficulties to fill in them adequately.

\section{Data collection}

All participating women completed a baseline data sheet in the practice with demographic details such as age, partnership, children and professional qualifications and a medical history including appearance and smell of urine, accompanying symptoms such as fever and duration of symptoms. Urine samples were tested for leucocytes, erythrocytes and nitrite in the practice and sent to the laboratory in charge to be tested for the type of germ, number and resistance profile.

Each woman was asked to complete the UTI-SIQ-8 questionnaire at inclusion and for 7 days consecutively. Besides, women completed the KHQ_G for assessing the quality of life in women with urinary incontinence, the EQ-5D, to measure the health-related quality of life and a VAS for rating pain. The questionnaires in the study were used for self-assessment either at inclusion only (KHQ_G) or also for the following 7 days (UTI-SIQ-8, VAS pain scale, EQ-5D).

Women could enter their data online via a web application for smartphones, smartwatches and tablets, including a gentle daily reminder to fill in the questionnaires. Online data collection was realised by a specifically programmed, responsive, dynamic web form. Data transmission was always ensured to be encrypted via SSL/ HTTPS. All personal data stored in the relational MySQL database was encoded via symmetric salted cryptography algorithms. All survey data were stored in separate data tables and allocated using only an anonymised participant ID. Moreover, regarding data export, the participant ID was additionally scrambled, rendering the matching of survey data to personal information practically impossible (see online supplementary appendix 2). It was also possible to use a paper-and-pencil version to ensure participation of those who were not able to use the mobile app.

While the electronically collected data could be transferred directly into the database, the nonelectronic data (paper-and-pencil version, urine test results) had to be entered by the study team manually.

\section{Statistical analysis}

The UTI-SIQ-8 was evaluated in seven steps.

\section{Item analysis}

Mean scores, SDs and ranges were analysed to evaluate item score and scale score distributions, especially to check for floor and ceiling effects.

\section{Reliability}

We determined Cronbach's alpha as a measure of internal consistency based on the intercorrelations among the test items.

\section{Convergent validity}

Convergent validity was assessed in terms of the strength of the associations between the UTI-SIQ-8 scores and the EQ-5D pain and activity scores and the VAS.

\section{Discriminant validity}

Discriminant validity was investigated by comparing UTISIQ-8 items and those EQ-5D and KHQ items that should measure distinct dimensions. For example, we expected that the KHQ incontinence item was only moderately correlated with the UTI-SIQ-8 total score.

\section{Factorial validity}

To explore the factorial structure and to check potential cross-loadings of the items, we ran a principal component analysis using Promax rotation, as we expected the influence of correlated factors. We used the scree plot, the Kaiser criterion and parallel analysis ${ }^{29}$ as criteria to decide how many factors to extract. However, because the scree plot criterion is somewhat subjective, and using the Kaiser criterion can render us susceptible to overestimating the number of factors, we used O'Connor' syntax $^{30}$ and ran a parallel analysis ${ }^{29}$ to determine objective and reliable indices for factor extraction. To test for unidimensionality, we additionally ran a confirmatory factor analysis using the $\mathrm{R}$ package lavaan. ${ }^{31}$ The model was estimated using maximum likelihood estimation. Model fit was assessed with the Comparative Fit Index (CFI), root mean square error of approximation (RMSEA) and standardised root mean square residual 
(SRMR) such that values of the $\mathrm{CFI} \geq 0.90$, RMSEA $\leq 0.08$ and SRMR $\leq 0.11$ are considered to reflect an acceptable fit to the data.

\section{Sensitivity to change}

To investigate sensitivity to change, we analysed whether changes across days in the UTI-SIQ-8 scores were associated with changes in quality of life (EQ-5D) and pain (VAS). To account for the hierarchical data structure, we used multilevel random coefficient modelling ${ }^{32}$ with the R package lme $4 .{ }^{33}$ Days (level 1 ) were nested within individuals (level 2). Two separate models were run to examine the covariability between the quality of life (pain) and symptom severity measured via UTI-SIQ-8. To improve the interpretability of the model results, the predictor variables were group mean-centred before running the models. We also determined the intraclass correlation (ICC) as an indicator of the sensitivity to change of the UTI-SIQ-8. This process was mandated to determine the degree of changes expected in the UTISIQ-8 scores, which could be attributed to differences across days versus differences among people.

\section{Network analysis}

To determine the relative position of UTI-SIQ-8 among other theoretically relevant constructs of symptom severity and to further establish convergent and discriminant validity, we examined distinctive associations between the total score of the UTI-SIQ- 8 and the measures of quality of life regarding autonomy and pain (both EQ-5D), pain (VAS) and impairment due to incontinence and impairment of personal relationships (both KHQ-G) by using network analysis. The novel approach of network analysis facilitates visualisation, description and significance testing of relations between variables while controlling for the influence of other variables. In network analysis, variables are called nodes and relations between variables are called edges. In the present analyses, we entered the UTI-SIQ-8 scale score, quality of life regarding autonomy and pain, pain (VAS), and impairment due to incontinence and impairment of personal relationships as the observed nodes of the network. Edges may be described as partial correlations controlling for all other relationships in the network $\left(r_{p}\right)$. Network analyses were conducted using the $\mathrm{R}$ packages qgraph ${ }^{34}$ and bootnet. ${ }^{33}$ The analyses followed suggestions from Epskamp and colleagues. ${ }^{35}$ To address potential difficulties of interpretation, we followed the conservative way of regularisation and interpretation.

\section{Patient and public involvement}

Patients and public were not involved in the planning and design of this study. All participants who were included in the study gave written informed consent after a thorough explanation of the procedures involved.

Ethics approval was obtained from the ethics committee of Göttingen Medical School (17/4/16).
RESULTS

\section{Patient characteristics}

A total of 18 practices with a total of 131 women took part in the study. We excluded 11 women from analysis due to screening failure or technical reasons, such as missing or erroneous data, resulting in a valid sample of 120 women with a total of 769 symptom reports across 7 days of measurement. The mean age of the women was $43.3 \pm 16.6$ years, the urine dipstick was positive for leucocytes in $92 \%$, erythrocytes in $87 \%$ and nitrites in $23 \%$, and the urine culture was positive in $82 \%$ (96/118). Escherichia coli was found in $78 \%(74 / 96)$ of the positive urine cultures. Detailed information on the patient characteristics is reported in Gágyor et al. ${ }^{28}$

The majority of the participating women $(87 / 120)$ used the web application via smartphone, smartwatches or tablets. In the patients 65 years and older, 12 women participated. Half of them $(6 / 12)$ chose the electronic version.

\section{Item analysis}

Table 1 shows the mean scores and SDs as well as further statistical characteristics of the eight items of the UTISIQ-8 for the first 2 days, as reported by the women. They made use of the full scale ( 1 to 5 ) for all items on both days (table 1), resulting in high SDs (1.0 to 1.79$)$. The coefficients for skewness ranged from 0.048 to 0.466 on day 0 , indicating a low level of skewness and were somewhat higher on day 1 . The mean scores strongly decreased from day 0 to day 1 , indicating a sharp decline in symptom severity and impairment caused by these symptoms (bothersomeness). The distribution of the items on the following days was more positively skewed as most patients substantially recovered within a few days.

\section{Reliability}

Cronbach's alpha for the eight items was high on day 0 (0.86) and even higher on day 1 (0.91), confirming the internal consistency of the UTI-SIQ-8. Notably, six items contributed equally to the reliability of the scale and only two (abdominal) pain items did not correlate as high as the other items with the total score (table 2).

\section{Validity}

Factorial validity

Preliminary results revealed one or three factors based on the conventional scree plot criterion and three factors based on the Kaiser criterion. A parallel analysis, which provides more objective and reliable indices for factor extraction, highlighted two factors (table 3). The twofactor solution explained $74 \%$ of total item variance and confirmed the special role of the two pain items as already detected by the corrected item-total correlations for the reliability analysis. While six of the items, referring to urgency, dysuria and frequency, loaded high on one factor, both pain items loaded on a second factor. However, since Cronbach's alpha was satisfactory for all eight items and all factors should consist of more 
Table 1 Distribution of responses to each UTI-SIQ-8 item

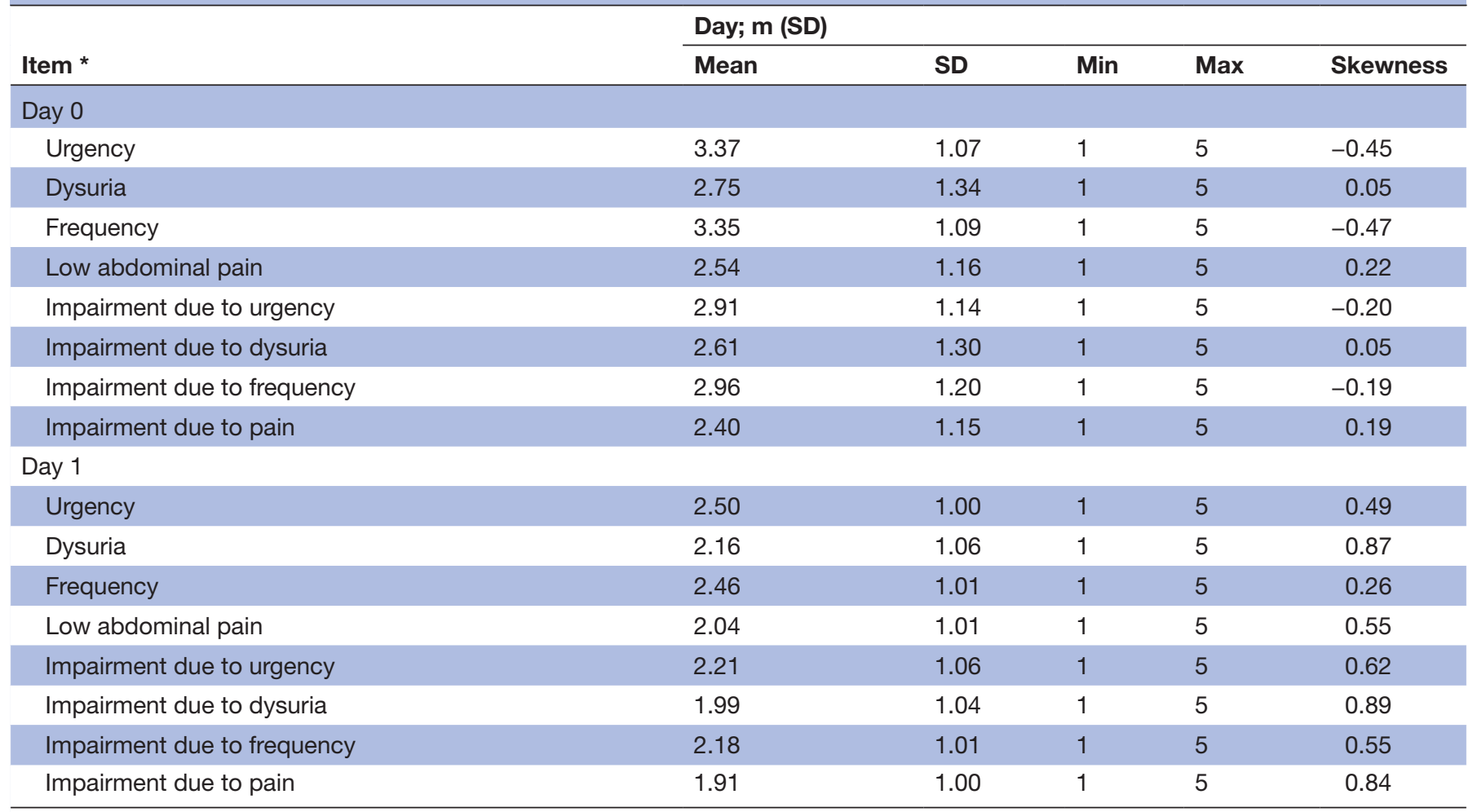

*Response rate ranged between $n=118$ and 119 on day 0 and was $n=112$ on day 1 .

UTI-SIQ, urinary tract infection-Symptom and Impairment Questionnaire.

than two items, we decided to use a one-factor solution as suggested by the scree plot criterion for the following analyses.

To test for unidimensionality, a confirmatory factor analysis supported the one-factor solution $(\mathrm{CFI}=0.922$, RMSEA $=0.16$, SRMR $=0.066$ ) with correlated residuals between the items severity of dysuria and impairment (bothersomeness) due to dysuria and the items severity of low abdominal pain and impairment (bothersomeness) due to low abdominal pain. The model fit according to CFI and SRMR was acceptable, although the RMSEA was somewhat above the cut-off value of 0.08 .

\section{Convergent validity}

The UTI-SIQ-8 was significantly and positively correlated with the pain and activity subscales of the EQ-5D and the VAS (table 4), confirming convergent validity. As expected, patients who reported stronger symptom severity and perceived impairment by UTI symptoms also reported more pain and an impairment of usual activities.

\section{Discriminant validity}

The correlations between the UTI-SIQ-8 and impairment due to incontinence as well as the activity subscale of the EQ-5D were smaller than the convergent validity correlations, exemplifying discriminant validity (table 4). As expected, our results indicated that women who reported more severe symptoms and impairment by UTI symptoms reported to a lesser degree that they suffered from incontinence but suffered more from pain and/or an impairment in usual activities. The correlation between reported symptom severity/impairment by UTI symptoms and impairment due to reduced mobility, self-care or anxiety/depression was also lower than the correlation with pain or an impairment in usual activities. Both results highlight discriminant validity.

\section{Sensitivity to change}

The ICC $=0.32$ indicates that $32 \%$ of the total variance in the UTI-SIQ-8 scores was due to differences between the women and $68 \%$ due to time differences. Differences between the item scores can be explained from daily changes in a person's symptoms, which highlights the sensitivity to change of the UTI-SIQ-8. Importantly, these daily changes in an individual's symptoms moved parallel with daily changes in the EQ-5D $(b=1.68, \mathrm{SE}=0.12$, $\mathrm{p}<0.001)$ and the VAS $(\mathrm{b}=0.03, \mathrm{SE}=0.003, \mathrm{p}<0.001)$, thus highlighting convergent validity with respect to daily changes in symptom severity.

\section{Network analysis}

Figure 1 visualises the estimated network of the UTISIQ-8. The UTI-SIQ-8 takes a central position between the VAS and some of the selected subscales of the EQ-5D and KHQ-G. Symptom severity, as assessed with the UTI-SIQ-8, exhibited a unique connection with pain as assessed with the VAS $\left(r_{p}=0.34\right)$ and, to a smaller extent, with impairment in personal relationships (KHQ-G; $r_{p}=0.25$ ) and 
Table 2 Reliability of the UTI-SIQ

\begin{tabular}{|c|c|c|}
\hline Items & Alpha & $\begin{array}{l}\text { Corrected } \\
\text { item total } \\
\text { correlation }\end{array}$ \\
\hline Day 0 & 0.86 & \\
\hline Urgency & & 0.71 \\
\hline Dysuria & & 0.63 \\
\hline Frequency & & 0.57 \\
\hline Low abdominal pain & & 0.44 \\
\hline Impairment due to urgency & & 0.76 \\
\hline Impairment due to dysuria & & 0.67 \\
\hline Impairment due to frequency & & 0.70 \\
\hline Impairment due to pain & & 0.39 \\
\hline Day 1 & 0.91 & \\
\hline Urgency & & 0.77 \\
\hline Dysuria & & 0.67 \\
\hline Frequency & & 0.74 \\
\hline Low abdominal pain & & 0.58 \\
\hline Impairment due to urgency & & 0.83 \\
\hline Impairment due to dysuria & & 0.74 \\
\hline Impairment due to frequency & & 0.83 \\
\hline Impairment due to pain & & 0.58 \\
\hline
\end{tabular}

${ }^{*}$ Response rate ranged between $n=118$ and 119 on day 0 and was $\mathrm{n}=112$ on day 1 .

UTI-SIQ, urinary tract infection-Symptom and Impairment Questionnaire.

usual activities and pain/discomfort (EQ-5D; $\left.\mathrm{r}_{\mathrm{p}}=0.20\right)$. Symptom severity (UTI-SIQ-8) was only slightly related to impairment due to incontinence (KHQ-G, $r_{p}=0.14$ ) and negatively related to mobility, self-care and anxiety/ depression (EQ-5D; $\left.\mathrm{r}_{\mathrm{p}}=-0.19\right)$.

\section{DISCUSSION}

The UTI-SIQ-8 proved to be a reliable and valid questionnaire to measure symptom severity and impairment (bothersomeness) and the symptom course in women with UTI in German primary care. Women made use of the full scale of the eight items to assess the severity of their symptoms and the resulting impairment. Reliability was high with a Cronbach's alpha of around 0.9; convergent and divergent validity were also satisfactory. The daily changes in a woman's assessment of her symptoms moved parallel to the EQ-5D scores and the VAS for pain, underlining the convergent validity of the questionnaire for sensitivity to change. Network analysis showed a central position of the UTI-SIQ-8 between other measures of pain and the quality of life questionnaires (KHQ_G and EQ-5D). The web application was the women's preferred form to complete the questionnaire online.
Table 3 Factorial validity of the UTI-SIQ-8

\begin{tabular}{lcc}
\hline & \multicolumn{2}{l}{ Item loadings } \\
\cline { 2 - 3 } Items & Factor 1 & Factor 2 \\
\hline Day 0* & & \\
\hline Urgency & 0.87 & -0.03 \\
\hline Dysuria & 0.66 & 0.17 \\
\hline Frequency & 0.88 & -0.25 \\
\hline Low abdominal pain & -0.03 & 0.96 \\
\hline Impairment due to urgency & 0.86 & 0.05 \\
\hline Impairment due to dysuria & 0.67 & 0.22 \\
\hline Impairment due to frequency & 0.88 & -0.06 \\
\hline Impairment due to pain & -0.07 & 0.97 \\
\hline Day 1† & & \\
\hline Urgency & 0.91 & -0.06 \\
\hline Dysuria & 0.76 & 0.00 \\
\hline Frequency & 0.87 & -0.04 \\
\hline Low abdominal pain & 0.01 & 0.97 \\
\hline Impairment due to urgency & 0.88 & 0.04 \\
\hline Impairment due to dysuria & 0.84 & -0.02 \\
\hline Impairment due to frequency & 0.86 & 0.09 \\
\hline Impairment due to pain & 0.01 & 0.97 \\
\hline
\end{tabular}

*Variance explained: $74 \%$.

†Variance explained: $79 \%$.

UTI-SIQ, urinary tract infection-Symptom and Impairment Questionnaire.

\section{Strengths and limitations}

This is a validation study of a short UTI questionnaire that comprised both symptom severity and resulting impairment as perceived by women at consultation and during the course of symptoms. The validation study was conducted separately from the REGATTA triall ${ }^{21}$ to mitigate the risk of a selection bias, which is more likely in an RCT. We used different validation methods to demonstrate all relevant forms of validity and to inform future users about applicability.

\begin{tabular}{|c|c|}
\hline Other instruments & Pearson's r (p) \\
\hline \multicolumn{2}{|l|}{ Convergent validity } \\
\hline Pain and usual activities (EQ-5D) & $0.51(<0.001)$ \\
\hline VAS pain & $0.54(<0.001)$ \\
\hline \multicolumn{2}{|l|}{ Divergent validity } \\
\hline Incontinence (KHQ_G) & $0.28(0.002)$ \\
\hline $\begin{array}{l}\text { Mobility, self-care, anxiety/ } \\
\text { depression (EQ-5D) }\end{array}$ & $0.23(0.12)$ \\
\hline
\end{tabular}

KHQ_G, King's Health Questionnaire, German version; UTI-SIQ, urinary tract infection-Symptom and Impairment Questionnaire; VAS, visual analogue scale. 


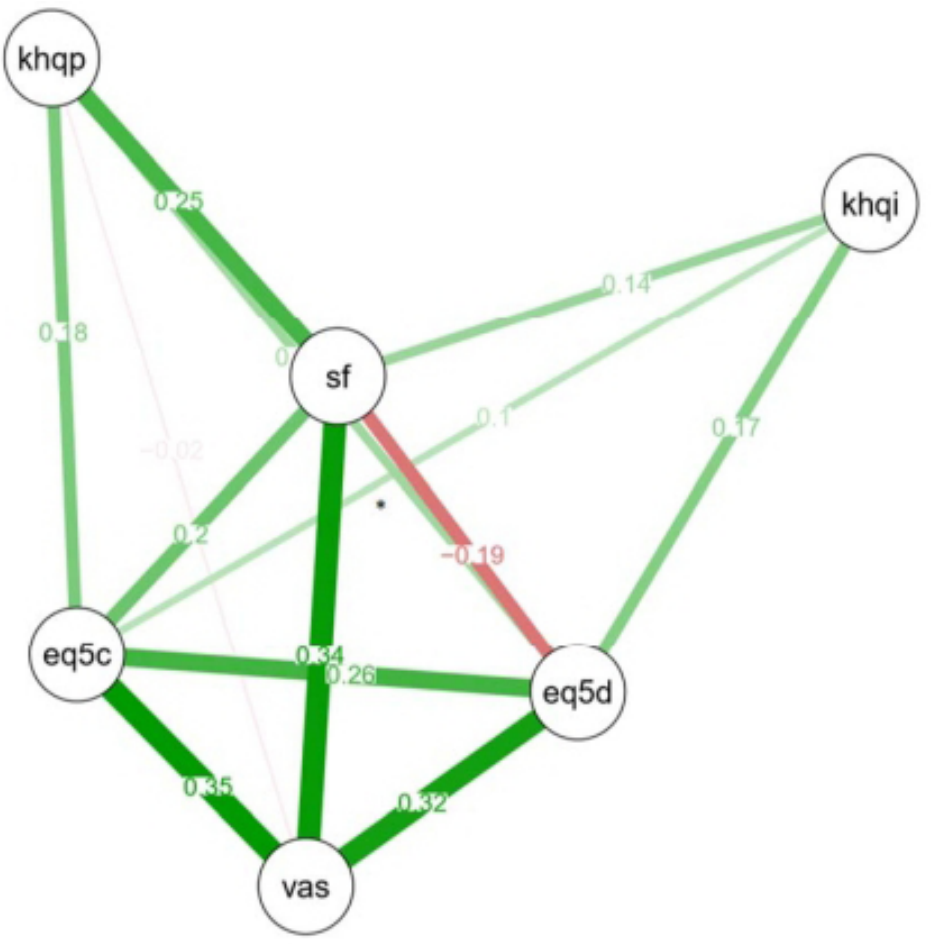

* Green lines indicate a positive connection; red lines indicate a negative connection. The saturation of the lines indicates
the strength of a connection.
Sf = UTI Symptom and Impairment Questionnaire (UTI-SIQ-8)
VAS = Visual Analog Scale for rating pain
eg5c = EQ-5D; pooled items usual activities, pain/discomfort
eg5d = EQ-5D; pooled items reduced mobility, self-care, and anxiety/depression
khqi = King's Health Questionnaire; scale "incontinence"
khqp = King's Health Questionnaire; scale "personal relations"

Figure 1 Network analysis.

Participating women had to complete a myriad of questionnaires but the UTI-SIQ-8 questionnaire is deliberately kept short to ensure a feasible implementation in daily use. There is some discussions about whether or not a shorter questionnaire lowers response burden and improves response rate. Rolstad $e t a l^{36}$ concluded in their review on response burden that there is a greater chance of response when patients were presented with a short questionnaire. They also argue that the quality of the content matters more from the patient's point of view than the length per se. However, since more items than absolutely necessary, daily or even more frequently asked, as other researches did, maybe a stress at least for some women, we consider the 8-item-survey an advantage of our study.

Our study was highly ecologically valid, as we followed patients in their daily life on a day-to-day basis. Because of the diary design of this study and the use of an online app, we were able to collect more than 750 data entries throughout the study highlighting the statistical power of our analyses. Using network analyses ${ }^{34}$ is novel in UTI research and may give direction to future research. For example, our research provided evidence that symptom severity, as assessed with the UTI-SIQ-8, takes a central position between the VAS and some of the selected subscales of the EQ-5D and KHQ-G supporting construct validity of our instrument.

Although the design of the study required only a small sample size of participating patients, the data suggest that the study population was comparable to other UTI studies in Germany. For example, a positive urine culture (approximately $75 \%$ ), prevalent among women with E. coli infections, and the susceptibility data were analogous with the results of other observational studies in Germany and RCTs. ${ }^{5-737-39}$

At present, we do not advise users to use the UTI-SIQ-8 for individual assessment because norm values or reference values are not yet available. Still, the questionnaire revealed satisfactory to good psychometric properties and it can thus be strongly recommended to use the UTISIQ-8 in research.

\section{Comparison with the existing literature}

Similar to the UTISA questionnaire, ${ }^{12}$ the UTI-SIQ-8 allowed women to rate the different UTI-related symptoms according to their severity and impairment (bothersomeness). In line with this study, we found that the severity and bothersomeness items loaded on the same 
factor. In other words, if a woman perceived a UTI symptom as severe, it also strongly affected her perceived impairment and vice versa; however, the authors of the UTISA decided after analysis to determine 'urination regularity' and 'problems with urination' as two different factors, as they did for pain symptoms. Our analysis inspired us to merge these items and we feel that the onefactor solution is logical from the patient perspective and allows a rapid and simple assessment of UTI symptoms and change across days.

Another self-reporting questionnaire, the ACSS, ${ }^{18}$ translated in many languages, also proved to be highly reliable and sensitive to change. While the 18-items questionnaire aims to assess the symptom severity and quality of life, in the first and the follow-up consultation, the UTI-SIQ-8 aims to assess symptom severity and bothersomeness on a day by day basis. This feature allows sensible monitoring of the symptom course as shown by several studies. ${ }^{6-8}$ Furthermore, these studies used a symptom diary, which was developed for RCTs to assess the UTI symptom at inclusion and to follow-up the symptom course and duration. The diary was validated for acute lower respiratory tract infections and showed good reliability and sensitivity to change but not for UTI. While it was unclear whether the results of the validation study are transferable to UTI patients, our analysis confirms the validity of this diary-based symptom assessment.

The items for low abdominal pain did not correlate as high as the other items with the total score of the UTISIQ-8, signalling a special role compared with the other symptoms. This finding corresponds to the results of Holm's et $a l^{15}$ validation study where the three items pain on urination, difficulties emptying the bladder and uncomfortable pressure around the bladder belonged to one dimension while a burning sensation on urination and pain around the bladder belonged to another dimension so that the authors concluded that patients must have perceived these as fundamentally different symptoms. We would not go so far but, indeed, abdominal pain seems only moderately linked with the remaining symptoms, namely, urgency, frequency and dysuria. Obviously not all women with UTI perceive abdominal pain, but nearly each woman with acute UTI experiences urgency, frequency and dysuria. ${ }^{1122}$ Since it is an important and troublesome symptom, as also Ferry et al. $^{11}$ suggested, we decided to leave the two pain items in the UTI-SIQ-8 but not as a factor of its own.

\section{Implications for practice and further research}

The UTI-SIQ-8 could be used for the baseline assessment of symptom severity in women with uncomplicated UTI and may help to decide whether or not to start with symptomatic treatment by using the delayed prescription approach. ${ }^{8}$ It can also be used to follow-up the course of the symptoms and their duration on a day by day basis to monitor treatment success and make changes in the treatment, if needed. A mobile health application could help to detect patients with worsening symptoms of complications, as demonstrated for other conditions. ${ }^{40}$ Further research is needed to identify the cut-off values for clinically relevant changes in the symptom course and duration.

As a side effect, the study indicates that web applications can be used for data collection via smartphones or other devices in all age groups, also for older patients.

\section{CONCLUSIONS}

The present findings support the UTI-SIQ-8 questionnaire as an economic, reliable and valid instrument for the assessment of symptom severity and symptom change in women with uncomplicated UTI. These findings may encourage primary care physicians to use it in their daily practice in order to gain more detailed information on the course of the condition. This may also support the communication and shared decision-making between physicians and patients. Researchers may apply the UTI-SIQ-8 to studies involving women with uncomplicated UTI.

\section{Author affiliations}

${ }^{1}$ Department of General Practice, Julius-Maximilians-Universitat Wurzburg, Wurzburg, Germany

${ }^{2}$ Department of General Practice/Family Medicine, University Medical Center Göttingen, Gottingen, Niedersachsen, Germany

${ }^{3}$ Psychological Assessment and Personality Psychology, Psychologische Hochschule Berlin, Berlin, Germany

${ }^{4}$ Department of Psychology, University of Göttingen, Göttingen, Germany

Acknowledgements The authors thank all participating women, all primary care investigators and their teams. Hannelore Schneider-Rudt helped organise the data. We also thank Professor Utz Reichard, Wagnerstibbe Laboratory (Amedes Group), Göttingen for the support of the study by providing the urine culture and sensitivity testing. Sven Wedeken designed the Web application for smartphones, smartwatches and tablets, and organised secure data transmission and data storage. We acknowledge support by the Open Access Publication Funds of the Göttingen University.

Contributors IG had the initial study idea; all authors worked out the final design of the study. SS-P collected the data. KR performed all statistical analyses. IG and WH were the major contributors in writing the manuscript and are the guarantors of the paper. All authors read, discussed and approved the final manuscript.

Funding The trial was funded solely by the German Federal Ministry of Education and Research (BMBF) Number: 01KG1105.

Competing interests None declared.

Patient consent for publication Not required.

Ethics approval The ethics committee of Göttingen Medical School approved the study (17/4/16). All participating physicians and patients provided written informed consent.

Provenance and peer review Not commissioned; externally peer reviewed.

Data availability statement Data are available upon reasonable request. The data used for the current study are available from the corresponding author on reasonable request.

Supplemental material This content has been supplied by the author(s). It has not been vetted by BMJ Publishing Group Limited (BMJ) and may not have been peer-reviewed. Any opinions or recommendations discussed are solely those of the author(s) and are not endorsed by BMJ. BMJ disclaims all liability and responsibility arising from any reliance placed on the content. Where the content includes any translated material, BMJ does not warrant the accuracy and reliability of the translations (including but not limited to local regulations, clinical guidelines, terminology, drug names and drug dosages), and is not responsible for any error and/or omissions arising from translation and adaptation or otherwise.

Open access This is an open access article distributed in accordance with the Creative Commons Attribution Non Commercial (CC BY-NC 4.0) license, which permits others to distribute, remix, adapt, build upon this work non-commercially, 
and license their derivative works on different terms, provided the original work is properly cited, appropriate credit is given, any changes made indicated, and the use is non-commercial. See: http://creativecommons.org/licenses/by-nc/4.0/.

\section{ORCID iDs}

Ildikó Gágyor http://orcid.org/0000-0002-7974-7603

Katrin Rentzsch http://orcid.org/0000-0001-8632-6842

Stephanie Strube-Plaschke http://orcid.org/0000-0002-1643-3025

Wolfgang Himmel http://orcid.org/0000-0003-3523-5486

\section{REFERENCES}

1 Butler CC, Hawking MKD, Quigley A, et al. Incidence, severity, help seeking, and management of uncomplicated urinary tract infection: a population-based survey. Br J Gen Pract 2015;65:e702-7.

2 Foxman B. The epidemiology of urinary tract infection. Nat Rev Urol 2010;7:653-60.

3 Heytens S, De Sutter A, De Backer D, et al. Cystitis: symptomatology in women with suspected uncomplicated urinary tract infection. $J$ Womens Health 2011;20:1117-21.

4 Leydon GM, Turner S, Smith $\mathrm{H}$, et al. Women's views about management and cause of urinary tract infection: qualitative interview study. BMJ 2010;340:c279.

5 Gágyor I, Bleidorn J, Kochen MM, et al. Ibuprofen versus fosfomycin for uncomplicated urinary tract infection in women: randomised controlled trial. BMJ 2015;351:h6544.

6 Vik I, Bollestad M, Grude N, et al. Ibuprofen versus pivmecillinam for uncomplicated urinary tract infection in women-A double-blind, randomized non-inferiority trial. PLoS Med 2018;15:e1002569.

7 Kronenberg A, Bütikofer L, Odutayo A, et al. Symptomatic treatment of uncomplicated lower urinary tract infections in the ambulatory setting: randomised, double blind trial. BMJ 2017;359:j4784.

8 Little P, Moore MV, Turner S, et al. Effectiveness of five different approaches in management of urinary tract infection: randomised controlled trial. BMJ 2010;340:c199.

9 Huttner A, Kowalczyk A, Turjeman A, et al. Effect of 5-day nitrofurantoin vs single-dose fosfomycin on clinical resolution of uncomplicated lower urinary tract infection in women: a randomized clinical trial. JAMA 2018;319:1781-9.

10 Wagenlehner FM, Abramov-Sommariva D, Höller M, et al. NonAntibiotic herbal therapy (BNO 1045) versus antibiotic therapy (fosfomycin trometamol) for the treatment of acute lower uncomplicated urinary tract infections in women: a double-blind, parallel-group, randomized, multicentre, non-inferiority phase III trial. Urol Int 2018;101:327-36.

11 Ferry SA, Holm SE, Stenlund H, et al. Clinical and bacteriological outcome of different doses and duration of pivmecillinam compared with placebo therapy of uncomplicated lower urinary tract infection in women: the LUTIW project. Scand J Prim Health Care 2007;25:49-57.

12 Clayson D, Wild D, Doll H, et al. Validation of a patient-administered questionnaire to measure the severity and bothersomeness of lower urinary tract symptoms in uncomplicated urinary tract infection (UTI): the UTI symptom assessment questionnaire. BJU Int 2005;96:350-9.

13 Wild DJ, Clayson DJ, Keating K, et al. Validation of a patientadministered questionnaire to measure the activity impairment experienced by women with uncomplicated urinary tract infection: the activity impairment assessment (AIA). Health Qual Life Outcomes 2005;3:42.

14 Fujimura T, Kume H, Tsurumaki Y, et al. Core lower urinary tract symptom score (CLSS) for the assessment of female lower urinary tract symptoms: a comparative study. Int J Urol 2011;18:778-84.

15 Holm A, Cordoba G, Siersma V. Development and validation of a condition-specific diary to measure severity, bothersomeness and impact on daily activities for patients with acute urinary tract infection in primary care. Health Qual Life Outcomes 2017;15:57.

16 Chang S-J, Lin C-D, Hsieh C-H, et al. Reliability and validity of a Chinese version of urinary tract infection symptom assessment questionnaire. Int. braz j urol. 2015;41:729-38.

17 Alidjanov JF, Pilatz A, Abdufattaev UA, et al. Deutsche Validierung des „Acute Cystitis Symptom Score“. Urologe 2015;54:1269-76.
18 Alidjanov J, Naber K, Abdufattaev U, et al. Reevaluation of the acute cystitis symptom score, a self-reporting questionnaire. Part I. development, diagnosis and differential diagnosis. Antibiotics 2018;7:6.

19 Homma Y, Yoshida M, Yamanishi T, et al. Core lower urinary tract symptom score (CLSS) questionnaire: a reliable tool in the overall assessment of lower urinary tract symptoms. Int J Urol 2008;15:816-20.

20 Pires IM, Marques G, Garcia NM, et al. A research on the classification and applicability of the mobile health applications. J Pers Med 2020;10. doi:10.3390/jpm10010011. [Epub ahead of print: 2702 2020].

21 Afshar K, Fleischmann N, Schmiemann G, et al. Reducing antibiotic use for uncomplicated urinary tract infection in general practice by treatment with uva-ursi (REGATTA) - a double-blind, randomized, controlled comparative effectiveness trial. BMC Complement Altern Med 2018;18:203.

22 Colgan R, Keating K, Dougouih M. Survey of symptom burden in women with uncomplicated urinary tract infections. Clin Drug Investig 2004;24:55-60.

23 Okamura K, Nojiri Y, Osuga Y. Reliability and validity of the King's health questionnaire for lower urinary tract symptoms in both genders. BJU Int 2009;103:1673-8.

24 Bjelic-Radisic V, Dorfer M, Tamussino K, et al. Der King's Fragebogen zur Erfassung der Lebensqualität von Patientinnen mit Harninkontinenz (deutsche Version). Geburtshilfe Frauenheilkd 2005;65:1042-50.

25 EuroQol Research Foundation. EQ-5D-5L user guide, 2019. Available: https://euroqol.org/publications/user-guides [Accessed 2020 Jan 10].

26 Karcioglu O, Topacoglu H, Dikme O, et al. A systematic review of the pain scales in adults: which to use? Am J Emerg Med 2018;36:707-14.

27 EXPATICA. The German healthcare system: a guide to healthcare in Germany, 2020. Available: https://www.expatica.com/de/healthcare/ healthcare-basics/german-healthcare-system-103359/ [Accessed 2020 Dec 3].

28 Gágyor I, Strube-Plaschke S, Rentzsch K, et al. Management of urinary tract infections: what do doctors recommend and patients do? an observational study in German primary care. BMC Infect Dis 2020;20:813.

29 Horn JL. A rationale and test for the number of factors in factor analysis. Psychometrika 1965;30:179-85.

30 O'Connor BP. SPSS and SAS programs for determining the number of components using parallel analysis and velicer's MAP test. Behav Res Methods Instrum Comput 2000;32:396-402.

31 Rosseel Y. lavaan : An R Package for Structural Equation Modeling. J Stat Softw 2012;48.

32 Raudenbush SW, Bryk AS. Hierarchical linear models: applications and data analysis methods. Thousand Oaks, Calif: Sage Publ, 2002.

33 Bates D, Mächler M, Bolker B, et al. Fitting Linear Mixed-Effects Models Using Ime4. J Stat Softw 2015;67.

34 Epskamp S, Cramer AOJ, Waldorp LJ, et al. qgraph : Network Visualizations of Relationships in Psychometric Data. J Stat Softw 2012;48.

35 Epskamp S, Borsboom D, Fried El. Estimating psychological networks and their accuracy: a tutorial paper. Behav Res Methods 2018;50:195-212.

36 Rolstad S, Adler J, Rydén A. Response burden and questionnaire length: is shorter better? A review and meta-analysis. Value Health 2011;14:1101-8.

37 Bleidorn J, Gágyor I, Kochen MM, et al. Symptomatic treatment (ibuprofen) or antibiotics (ciprofloxacin) for uncomplicated urinary tract infection?--results of a randomized controlled pilot trial. BMC Med 2010;8:30.

38 Klingeberg A, Noll I, Willrich N, et al. Antibiotic-Resistant E. coli in uncomplicated community-acquired urinary tract infection. Dtsch Arztebl Int 2018;115:494-500.

39 Schmiemann G, Gágyor I, Hummers-Pradier E, et al. Resistance profiles of urinary tract infections in general practice--an observational study. BMC Urol 2012;12:33.

40 Sim I. Mobile devices and health. N Engl J Med 2019;381:956-68. 